\title{
Exoplanet atmospheres: A theoretical outlook
}

\author{
Sara Seager ${ }^{1}$ \\ ${ }^{1}$ Department of Earth, Atmospheric, and Planetary Sciences, Department of Physics, \\ Massachusetts Institute of Technology, Cambridge, Massachusetts, 02139 \\ email, seager@mit.edu
}

\begin{abstract}
With over two dozen exoplanet atmospheres observed today, the field of exoplanet atmospheres is solidly established. The highlights of exoplanet atmosphere studies include: detection of molecular spectral features; constraints on atmospheric vertical temperature structure; detection of day-night temperature gradients; and a new numerical approach to atmosphere temperature and abundance retrieval. As hot Jupiter observations and interpretation are maturing, the next frontier is super Earth atmospheres. Theoretical models of super Earth atmospheres are moving forward with observational hopes pinned on the James Webb Space Telescope, scheduled for launch in 2014. Further in the future lies direct imaging attempts to answer the enigmatic and ancient question, "Are we alone?" via atmospheric biosignatures.
\end{abstract}

Keywords. astrobiology, planetary systems, techniques: photometric

\section{Introduction}

At the dawn of the first discovery of exoplanets orbiting sun-like stars in the mid1990s, few believed that observations of exoplanet atmospheres would ever be possible. After the 2002 Hubble Space Telescope detection of a transiting exoplanet atmosphere, many skeptics discounted it as a one-object, one-method success. By 2010, the field was firmly established, with over two dozen exoplanet atmospheres observed today. Hot Jupiters. the type of exoplanet most amenable to study are observed by the dozens. Highlights include, detection of molecular spectral features; observation of day-night temperature gradients; and constraints on vertical atmospheric structure. Atmospheres of giant planets far from their host stars are also being studied with direct imaging. The ultimate exoplanet goal is to answer the enigmatic and ancient question, "Are we alone?" via detection of atmospheric biosignatures. The two paths forward are the nearterm focus on transiting super Earths orbiting in the habitable zone of M-dwarfs, and ultimately the space-based direct imaging of true Earth analogs.

\section{Past: A Brief History of Exoplanet Atmospheres}

The dawn of the discovery of exoplanets orbiting sun-like stars took place in the mid 1990s, with the birth of radial velocity detections. Because of selection effects, many of the exoplanets found in the first few years of discovery orbited extremely close to their host star. Called hot Jupiters, these planets orbit many times closer to their star than Mercury does to our sun. With semi-major axes 0.05 AU, the hot Jupiters are heated externally by their stars to temperatures of 1000-2000 K, or even higher. From the start the high temperature and close stellar proximity of hot Jupiters were recognized as favorable for atmospheric detection (Seager \& Sasselov 1998). Even as the number of exoplanet 


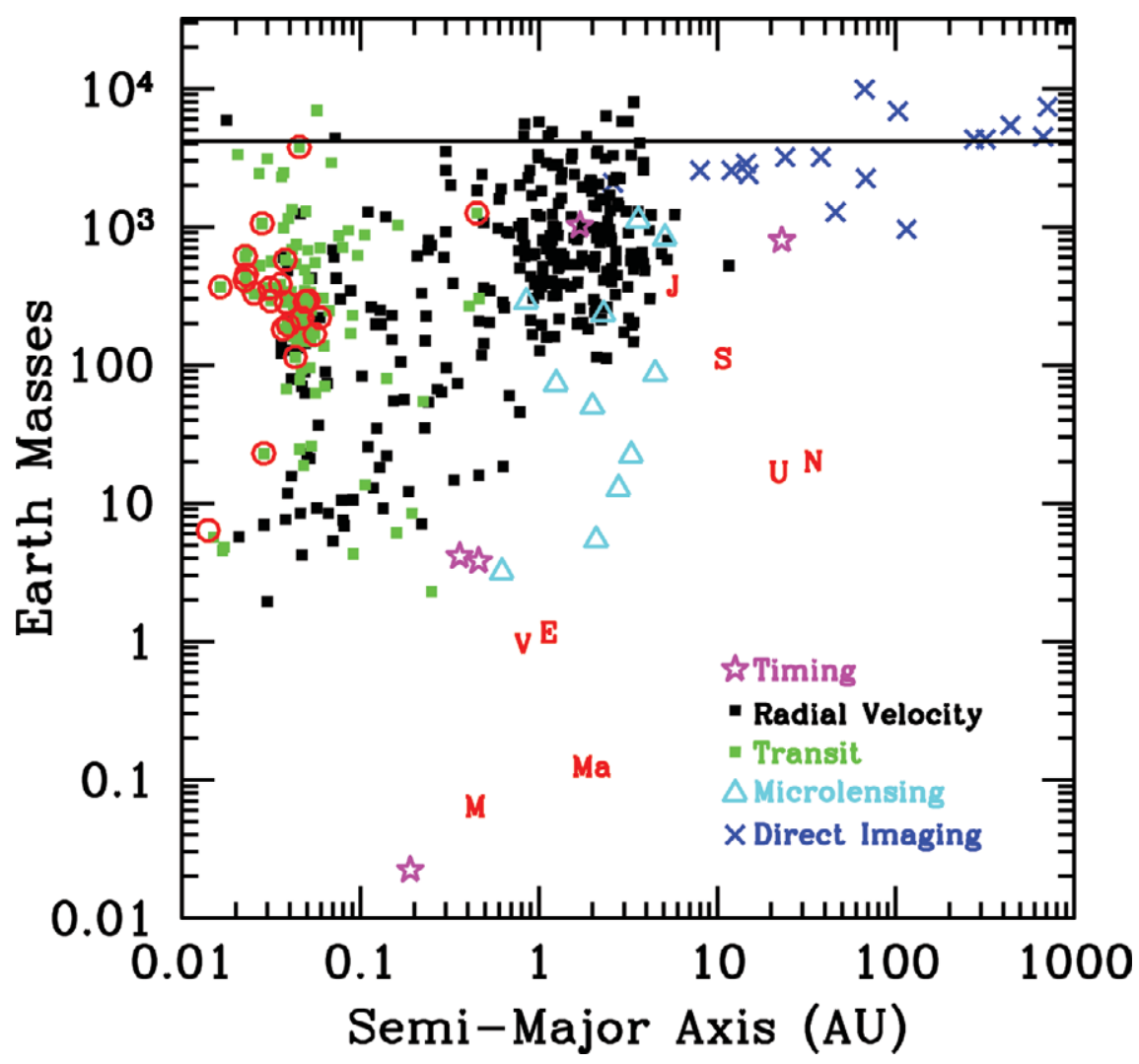

Figure 1. Known planets as of March 2010. Red letters indicate solar system planets. The red circles represent planets with published atmosphere detections. The solid line is the conventional upper mass limit for the definition of a planet. Data taken from http://exoplanet.eu/

detections grew in the late 1990 s (just under 30 by the end of the $20^{\text {th }}$ century $\dagger$ ), few thought that exoplanet atmospheres could be observed at any time in the foreseeable future.

By the time about seven hot Jupiters were known, the community expected one to transit. With a probability to transit of $R_{\star} / a$, where $R_{\star}$ is the stellar radius and $a$ is the semi-major axis, each hot Jupiter has about a $10 \%$ chance to transit. Seager \& Sasselov (2000) presented transit transmission spectra as a way to detect the atmospheres of hot Jupiters, by way of atomic and molecular transmission spectral features, with a focus on sodium. HD 209458b was found to show transits at the end of 1999 (Charbonneau et al. 2000; Henry et al. 2000), and the first detection of an exoplanet atmosphere, via atomic sodium, with the Hubble Space Telescope soon followed (Charbonneau et al. 2002).

The theory of exoplanet atmospheres was also developing in the 1990s and early 2000s. At that time, theory was leading observation, and observers consulted the model predictions to help define the most promising detection techniques. Most theory papers focused on irradiated hot Jupiters, emphasizing spectral features and 1D temperature/pressure profiles resulting from the intense heating by the host star (Seager \& Sasselov 1998; Marley et al. 1999; Sudarsky, Burrows, \& Pinto 2000; Barman, Hauschildt \& Allard 2001. Cloud modeling (Ackerman \& Marley 2001; Cooper et al. 2003) and atmospheric 
circulation (Showman \& Guillot 2002; Cho et al. 2003) were also expected to be important. Calculation of exoplanet illumination phase curves, polarization curves (Seager, Whitney, \& Sasselov 2000), and especially transmission spectra (Seager \& Sasselov 2000; Brown 2001; Hubbard et al. 2001) set the stage for observed spectroscopy during transit.

The Spitzer Space Telescope, launched in August 2003 revolutionized exoplanet atmosphere observations and hence theoretical modeling for interpretation. At mid-infrared wavelengths, hot Jupiters have a high planet-to-star contrast ratio, and the star and planet typically are bright enough to allow high precision photon-limited measurements. A flood of secondary eclipse observational detections came from Spitzer since 2005. Now hundreds of exoplanets are known (Figure 1). Dozens of hot Jupiters have been observed, creating the field of exoplanet atmospheres.

Here we summarize exoplanet atmosphere highlights then enumerate future prospects. For a full review, see Seager \& Deming (2010).

\section{Present: Recent Highlights}

Hot Jupiters dominate recent exoplanet atmosphere science, because their large radii, extended atmospheric scale heights, and high temperetures make atmosphere measurements possible.

\subsection{Hot Jupiters are Hot and Dark}

Hot Jupiters are blasted with radiation from the host star and thus should be kinetically hot. The first and most basic conclusion from the Spitzer secondary eclipse detections was the confirmation of this basic paradigm. The fact that the planets emit generously in the infrared implies that they efficiently absorb visible light from their stars. Searches for the reflected component of their energy budget have indicated that the planets must be very dark in visible light, with geometric albedos less than about 0.2 (Rowe et al. 2008), and likely much lower. Models show that purely gaseous atmospheres lacking reflective clouds will be very dark (Marley et al. 1999; Seager, Whitney, \& Sasselov 2000).

\subsection{Identification of Atoms and Molecules}

A major achievement for exoplanet atmospheres is the identification of atoms and molecules. In hot Jupiter atmospheres, the atoms and molecules identified are atomic sodium (Na) (e.g., Charbonneau et al. 2002), water vapor $\left(\mathrm{H}_{2} \mathrm{O}\right)$ (e.g., Swain et al. 2008; Figure 2), methane $\left(\mathrm{CH}_{4}\right)$ (Swain et al. 2008), carbon monoxide (CO), and carbon dioxide $\left(\mathrm{CO}_{2}\right)$ (e.g., Swain et al. 2009a,b; Madhusudhan and Seager 2009). (For a critical discussion of Swain et al. 2008, see Gibson et al. 2011 and Swain et al. this volume.) In addition to molecules, the presence of atmospheric haze has been inferred in HD 189733 via transmission spectra with HST/STIS. While the particle composition has not been identified, the Rayleigh-scattering behavior of the data indicates small particle sizes (Pont et al. 2008). A thorough temperature and abundance retrieval method enables statistical constraints on molecular mixing ratios and other atmospheric properties (see Fig. 3 and Madhusudhan \& Seager, 2009).

\subsection{Day-Night Temperature Gradients}

Hot Jupiters are theorized to have their rotation synchronized with their orbital motion by tidal forces, a process that should conclude within millions of years (e.g., Guillot et al. 1996). Under this tidal-locking condition the planet will have a permanent day side and a permanent night side. Spitzer thermal infrared observations of HD 189733b shows that the planet has only a moderate temperature variation from the day to night side. The 


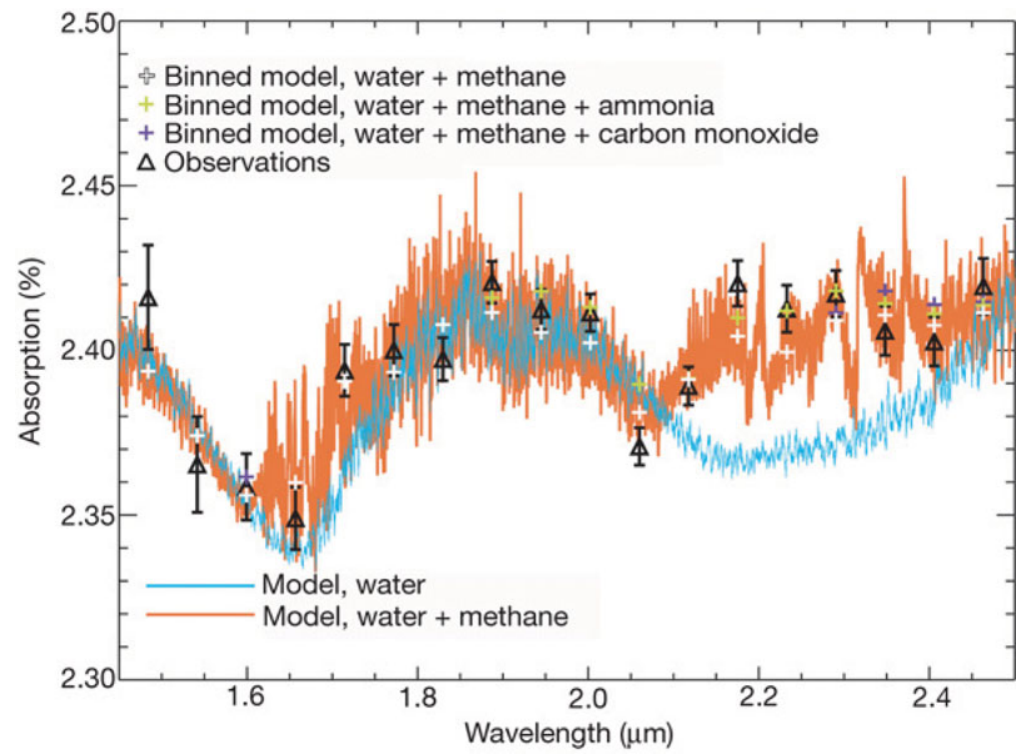

Figure 2. Transmission spectrum of the transiting planet HD 189733. Hubble Space Telescope observations shown by the black triangles. Two different models highlight the presence of methane in the planetary atmosphere. From Swain et al. (2008).
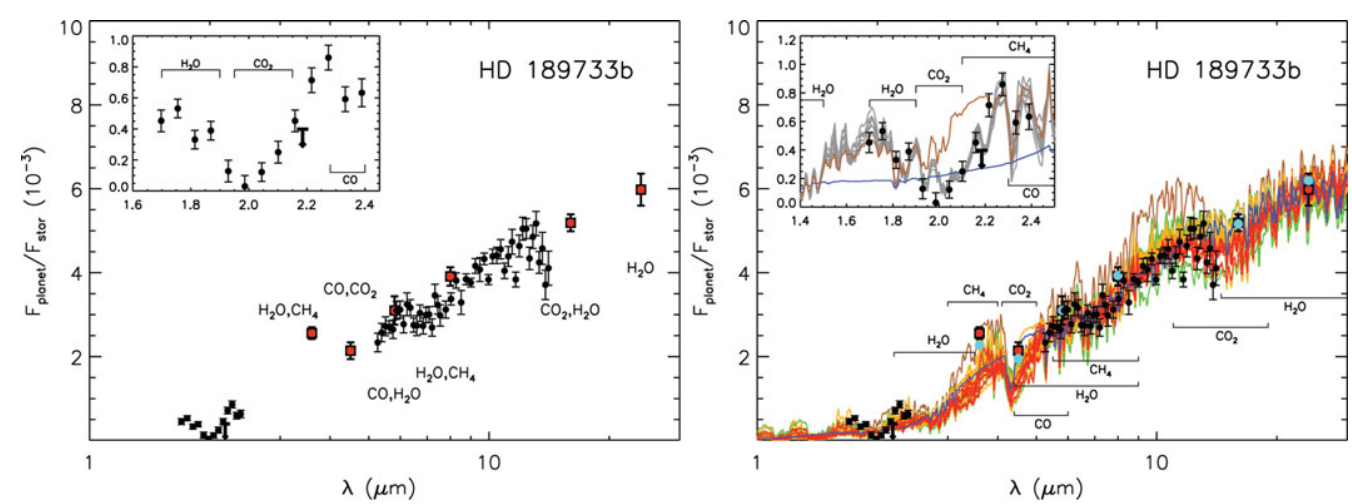

Figure 3. Thermal emission data composite for HD 189733 in secondary eclipse. Data from HST/NICMOS (inset, Swain et al. 2009), Spitzer/IRAC (four shortest wavelength red points; Charbonneau et al. 2008), Spitzer/IRS-PU (Deming et al. 2006), Spitzer/MIPS (Charbonneau et al. 2008), Spitzer/IRS (black points from $5-13 \mu \mathrm{m}$; Grillmair et al. 2008). Models shown in the right panel (from Madhusudhan and Seager 2009) illustrate that the best fits to the Spitzer/IRS ((red curve shows fits within the $1.4 \sigma$ errors, on average; orange $1.7 \sigma$, green $2 \sigma$, and blue is one best fit model within $1.4 \sigma$ ) and Spitzer photometry (brown curve within $1 \sigma$ ) do not fit the NICMOS data (inset grey curves within $1.4 \sigma$ ) possibly implying variability in the planet atmosphere from data taken at different epochs. For abundance constraints from the different models, see Madhusudan and Seager (2009).

planet shows an $8 \mu \mathrm{m}$ brightness temperature variation of over $200 \mathrm{~K}$ from a minimum brightness temperature of $973 \pm 33 \mathrm{~K}$ to a maximum brightness temperature of $1212 \pm 11 \mathrm{~K}$ (Knutson et al. 2007), and a thermal brightness change at $24 \mu \mathrm{m}$ consistent with the $8 \mu \mathrm{m}$ data within the errors (Knutson et al. 2009). Model interpretation indicates that strong winds have advected the hottest region to the east of the sub-stellar point (Knutson 


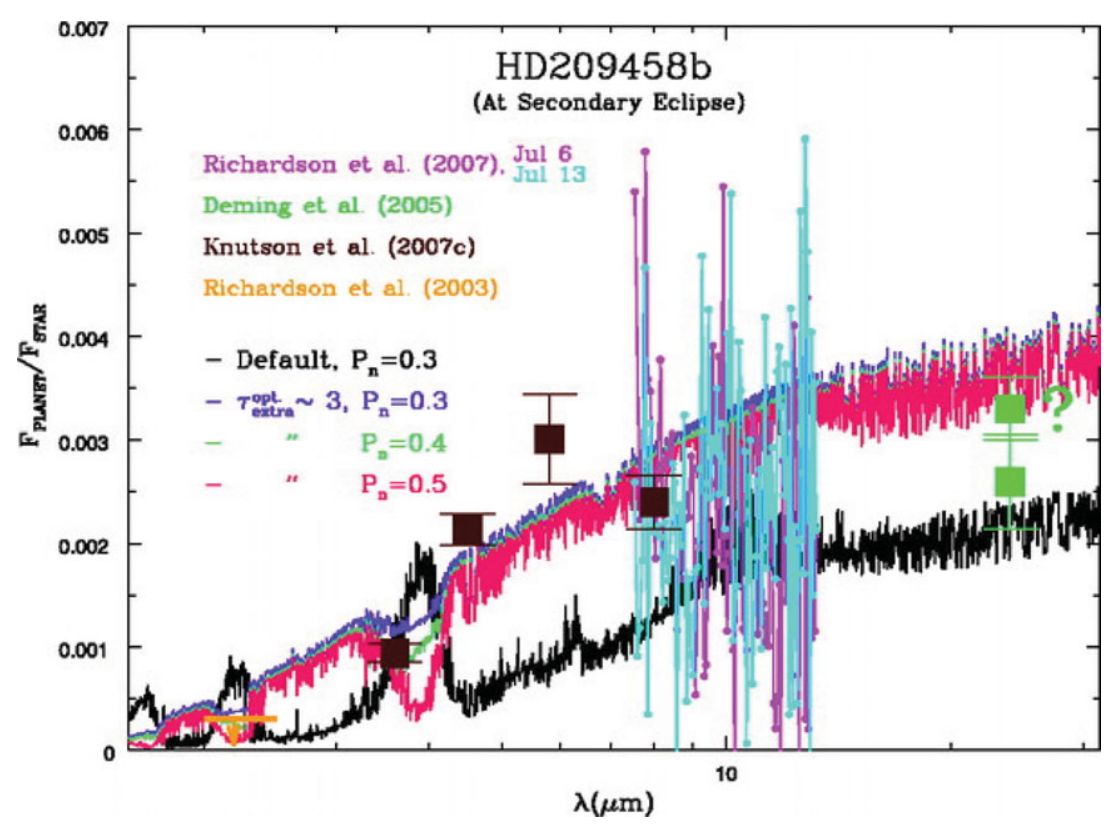

Figure 4. Evidence for an atmospheric thermal inversion for HD 209458b. Spitzer data points from secondary eclipse measurements are shown with brown (IRAC; Knutson et al. 2007) and green (Deming et al. 2005 and private comm.; the two points are data taken at different times). IRS spectra shown in purple and aqua are from Richardson et al. (2007). The model in pink shows emission features from an atmospheric thermal inversion. The black curve is a non-thermal-inversion model. Figure from Burrows et al. (2007).

et al. 2007; Showman et al. 2009). The shifted hot region on the dayside carries physical information such as the speed of the zonal circulation, and information about the altitude and opacity-dependence of the atmospheric radiative time constant.

\subsection{Atmospheric Escape}

Escaping atomic hydrogen from the exosphere of the hot Jupiter HD 209458b has been detected during transit in the Ly $\alpha$ line. A positive detection was made with HST/STIS $(3.75 \sigma)$ (Vidal-Madjar et al. 2003). Showing a 15\% drop in stellar Ly $\alpha$ intensity during transit, the HD 209458b observations are interpreted as a large cloud of hot hydrogen surrounding the planet. The cloud extends up to four planetary radii, and the kinetic temperature is as high as tens of thousands of K. Models agree that the implied exospheric heating is likely due to absorption of UV stellar flux, but Jeans escape is not sufficient to account for the hydrogen cloud. The specific origin of the escaping atoms is modeldependent. Escape mechanisms include radiation pressure, charge exchange, and solar wind interaction (see, e.g., Lammer et al. 2009 and references therein).

\subsection{Vertical Thermal Inversions}

Evidence for vertical atmospheric thermal inversions in hot Jupiters comes from emission features in place of (or together with) absorption features in the thermal infrared spectrum (for a basic explanation see Seager 2010). Because broad-band photometry does not delineate the structure of molecular spectral bands, the inference of a thermal inversion must rely on models. Spitzer data show that the upper atmospheres of several planets have thermal inversions, if water vapor is present and if abundances are close to solar (e.g., Burrows et al. 2008; see Figure 4). 
The hot Jupiter temperature inversions are likely created by absorption of stellar irradiance in a high-altitude absorbing layer. Madhusudhan \& Seager (2010), however, have found that for many cases existing observations (Spitzer broad-band photometry) are not enough to make robust claims on the presence of thermal inversions.

\section{Future Prospects}

\subsection{Super Earth Atmospheres}

In exoplanet research the frontier is always the most exciting. In exoplanet atmospheres, the frontier is the field of super Earths. Super Earths are unofficially defined as planets with masses between 1 and 10 Earth masses. The term super Earths should define planets that are rocky in Nature, rather than planets with icy interiors or significant gas envelopes. Because there may be a continuous and overlapping mass range between them, super Earths and "exo-Neptunes" are often discussed together.

The major challenge to studying super Earth atmospheres, is the anticipated wide diversity. This is different from Jupiter and the other solar system gas giants, which have "primitive" atmospheres. That is, Jupiter has retained the gases it formed with, and these gases approximately represent the composition of the sun. The super Earth atmospheres, in contrast, could have a wide range of possibilities for the atmospheric mass and composition. Attempts to evaluate these possibilities used calculations of atmospheres that formed by outgassing during planetary accretion, considering bulk compositions drawn from differentiated and/or primitive solar system meteoritic compositions (Elkins-Tanton \& Seager 2008; Schaefer \& Fegley 2010). Instead of narrowing down possibilities, this work emphasized the large range of possible atmospheric mass and composition of outgassed super Earths even before consideration of atmospheric escape.

Researchers take different paths for modeling super Earth atmospheres, focusing on different regions of parameter space. One approach is to consider atmospheres similar to Earth, Venus or Mars (or their atmospheres in earlier epochs). Considering the amount of greenhouse gases including $\mathrm{CO}_{2}$, Selsis et al. (2007) and von Bloh et al. (2007) both found that Gl 581d is more likely to be habitable (that is with surface temperatures consistent with liquid water) than Gl 581c. Other investigators consider atmospheres that radically depart from the terrestrial planets in our solar system. Water planets, akin to scaled up versions of Jupiter's icy moons, could have up to 50 percent water by mass, with concomitant massive steam atmospheres (Kuchner 2003; Leger et al. 2004; Rogers \& Seager 2010). In a different approach, Miller-Ricci, Seager, \& Sasselov (2009) considered GJ 581c and three possibilities relating to atmospheric hydrogen content. A suggestion of terrestrial planets with sulfur cycles dominating over carbon cycles is described in Kaltenegger \& Sasselov (2010). Others have attempted to quantify the atmospheric escape of Earths and super Earths, with little success due to the unknown initial mass and star's activity history (e.g., Lammer et al. 2007).

We anticipate the discovery of a handful of rare but highly valuable transiting super Earths in the habitable zones of the brightest low-mass stars. With such targets, we will observe the transiting super Earth atmospheres in the same way we are currently observing transiting hot Jupiters orbiting sun-like stars. NASA's JWST scheduled for launch in 2014, will be capable of observing the absorption signatures of major molecules like water and carbon dioxide. Such observations will require monitoring of multiple transits, often amounting to $\sim 100$ hours of JWST observation (Deming et al. 2009). 

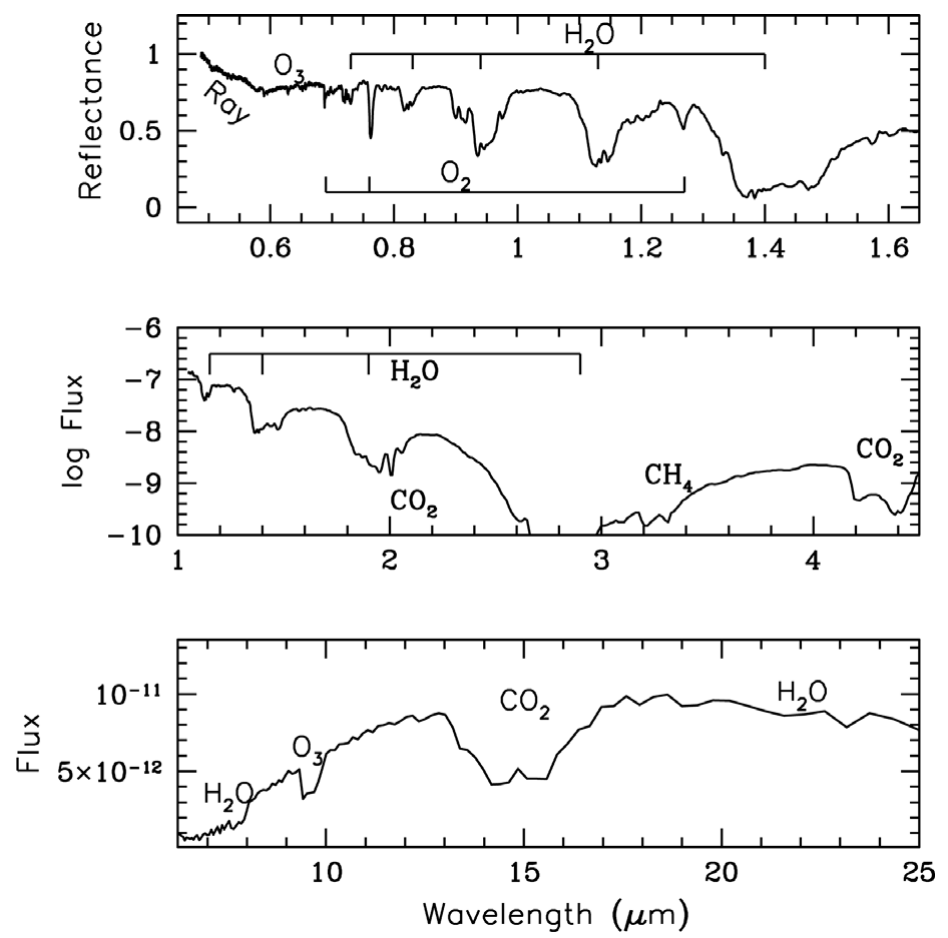

Figure 5. Earth as an exoplanet: Earth's hemispherically averaged spectrum. Top: Earth's visible wavelength spectrum from Earthshine measurements plotted as normalized reflectance (Turnbull et al. 2006). Middle: Near-infrared spectrum from NASAs EPOXI mission with flux in units of $\mathrm{W} \mathrm{m}{ }^{2}$ ? $\mathrm{m}^{1}$ (Robinson et al., 2010). Bottom: Earth's mid-infrared spectrum as observed by Mars Global Surveyor enroute to Mars with flux in units of $\mathrm{W} \mathrm{m}^{2} \mathrm{~Hz}^{1}$ (Pearl and Christensen 1997). Major molecular absorption features are noted including Rayleigh Scattering. Only strongly absorbing, globally mixed molecules are detectable.

\subsection{Earth Analog Atmospheres and Biosignature Gases}

Without question the holy grail of exoplanet research is the discovery of a true Earth analog, an Earth-size, Earth-mass planet in an Earth-like orbit about a sun-like star. We emphasize that discovery of Earth-size or Earth-mass planets-even those in their star's habitable zone-is not the same as identifying a habitable planet. Venus and Earth are both about the same size and mass-and would appear the same to an astrometry, radialvelocity, or transit observation. Yet Venus is completely hostile to life due to the strong greenhouse effect and resulting high surface temperatures (over $700 \mathrm{~K}$ ), while Earth has the right surface temperature for liquid water oceans and is teeming with life. This is why, in the search for habitable planets, a direct-imaging space-based telescope capable of blocking out the starlight is inevitable.

The main motivation for finding Earth analogs is to search their atmospheric spectra for biosignature gases. An atmospheric biosignature gas is one produced by life. The canonical concept for the search for atmospheric biosignatures is to find an atmosphere severely out of thermochemical redox equilibrium (Lederberg 1965; Lovelock 1965). Indeed Earth's atmosphere has oxygen (a highly oxidized species) and methane (a very reduced species) several orders of magnitude out of thermochemical redox equilibrium.

In practice it could be difficult to detect molecular features from different redox states. The Earth as an exoplanet, for example (Figure 5), has a relatively prominent oxygen 


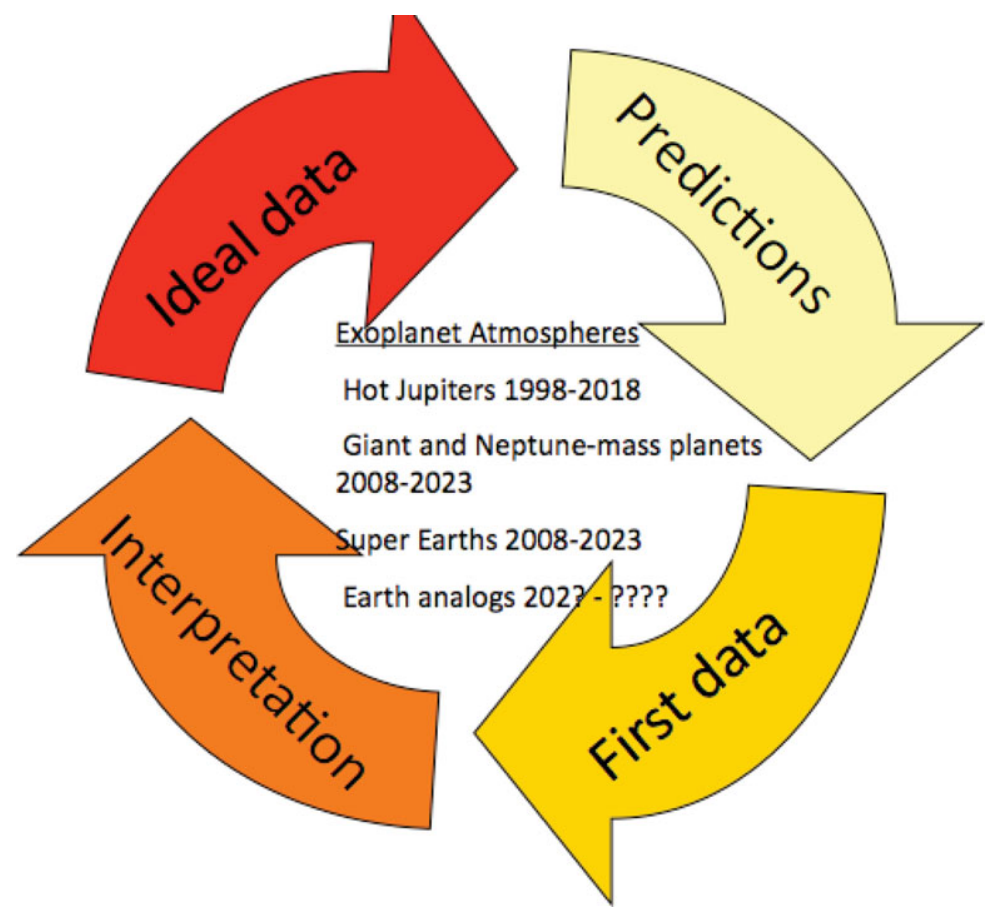

Figure 6. Cycles for exoplanet atmospheres.

absorption feature at $0.76 \mu \mathrm{m}$, whereas methane at present-day levels of $1.6 \mathrm{ppm}$ has only extremely weak spectral features. The more realistic atmospheric biosignature gas is a single gas completely out of chemical equilibrium. Earth's example again is oxygen or ozone, about ten orders of magnitude higher than expected from equilibrium chemistry and with no known abiotic production at such high levels. The challenge with a single biosignature outside of the context of redox chemistry becomes one of false positives. To avoid false positives we must look at the whole atmospheric context.

Most biosignatures work to date has focused on mild extensions of exoplanet biosignatures as on Earth $\left(\mathrm{O}_{2}, \mathrm{O}_{3}, \mathrm{~N}_{2} \mathrm{O}\right.$ ) or early Earth (possibly $\mathrm{CH}_{4}$ ) biosignatures. Research forays into biosignature gases that are negligible on Earth but may play a more dominant role on other planets has started. Pilcher (2003) suggested that organosulfur compounds, particularly methanethiol $\left(\mathrm{CH}_{3} \mathrm{SH}\right.$, the sulfur analog of methanol) could be produced in high enough abundance by bacteria, possibly creating a biosignature on other planets. Pilcher (2003) emphasized a potential ambiguity in interpreting the $9.6 \mu \mathrm{m} \mathrm{O} 3$ spectral feature since a $\mathrm{CH}_{3} \mathrm{SH}$ feature overlaps with it. Segura et al. (2005) showed that the Earth-like biosignature gases $\mathrm{CH}_{4}, \mathrm{~N}_{2} \mathrm{O}$, and even $\mathrm{CH}_{3} \mathrm{Cl}$ have higher concentrations and therefore stronger spectral features on planets orbiting M stars compared to Earth. The reduced UV radiation on quiet $\mathrm{M}$ stars enables longer biosignature gas lifetimes and therefore higher concentrations to accumulate. Seager, Schrenk, \& Bains (2010) have reviewed Earth-based metabolism to summarize the range of gases and solids produced by life on Earth. A fruitful new area of research will be on which molecules are potential biosignatures and which can be identified as such on super Earth planets different from Earth. 


\section{Outlook}

The field of exoplanet atmospheres is firmly established with a set of hot Jupiter observations and interpretation as the foundation. We see a cycle for atmospheric studies (Figure 6) that begins with predictions at a time when observations are leading theory. Next in the cycle comes the first truly breakthrough observations that enable a flurry of further observations. Third comes a period of interpretation or perhaps more aptly termed retrodiction, modeling and theory work that may raise more questions than answers and beg for better data; this part of the cycle is where observations are leading theory. We are at this point with hot Jupiter atmospheres. Closing the cycle comes when higher $\mathrm{S} / \mathrm{N}$ and higher spectral resolution data become available to answer the outstanding questions and provide closure. We foresee this closure for transiting hot Jupiters with future JWST observations.

With the cycle picture in mind we envision four eras for exoplanet atmospheric studies. The first is the hot Jupiter studies, with the start of the cycle in 1998 and with at least some closure by five years after JWST launch. The second era is that of more orbitally-distant giant planets and Neptunes, beginning now with direct imaging of young, hot Jupiters far from their stars, maturing with the next generation Gemini and VLT instrumentation, and finding some closure with the very large ground-based telescopes TMT/GMT/ELT of the future. The third era is also just beginning, that of transiting super Earths and mini Neptunes. With large amounts of JWST time the era of super Earths will advance to the third step of the exoplanet atmospheric cycle. The fourth era is that of true Earth analogs. Predictions using Earth as an exoplanet and some extensions are underway, but the first observations will have to await a specialized space telescope that can block out the orders of magnitude brighter starlight.

Thousands of years from now, people will look back and see as one of the most significant, positive accomplishments of our early twenty first century society the first discoveries of exoplanets, and, the human foray into finding and characterizing habitable planets. Exoplanet atmospheres plays a critical component as the hosts of signs of life on other worlds.

\section{References}

Ackerman, A. S. \& Marley, M. S. 2001, ApJ, 556, 872

Barman, T. S., Hauschildt, P. H., \& Allard, F. 2001, ApJ, 556, 885

Brown, T. M. 2001, ApJ, 553, 1006

Burrows, A., Budaj, J., Hubeny, I. 2008, ApJ, 678, 1436

Charbonneau, D., Brown, T. M., Latham, D. W., et al. 2000, ApJ, 529, L45

Charbonneau, D., Brown, T. M., Noyes, R. W., et al. 2002, ApJ, 568, 377

Charbonneau, D., Knutson, H. A., Barman, T., et al. 2008, ApJ, 686, 1341

Cho, J. Y.-K., Menou, K., Hansen, B. M. S., et al. 2003, ApJ, 587, 117

Cooper, C. S., Sudarsky, D., Milsom, J. A., et al. 2003, ApJ, 586, 1320

Deming, D., Seager, S., Richardson, L. J., et al. 2005, Nature, 434, 740

Deming, D., Harrington, J., Seager, S., et al. 2006, ApJ, 644, 560

Deming, D., Seager, S., Winn, J., et al. 2009, PASP, 121, 952

Elkins-Tanton, L. \& Seager, S. 2008, ApJ, 685, 1237

Gibson, N. P., Pont, F., \& Agrain, S. 2011, MNRAS, 411, 2199

Grillmair, C. J., Burrows, A., Charbonneau, D., et al. 2008, Nature, 456, 767

Guillot, T., Burrows, A., Hubbard, W. B., et al. 1996, ApJ, 459, L35

Henry, G. W., Marcy, G. W., Butler, R. P., et al. 2000, ApJ, 529, L41

Hubbard, W. B., Fortney, J. J., Lunine, J. I., et al. 2001, ApJ, 560, 413

Kaltenegger, L. \& Sasselov, D. 2010, ApJ, 708, 1162 
Knutson, H. A. Charbonneau, D., Noyes R. W., et al. 2007, ApJ, 655, 564

Knutson, H. A., Charbonneau, D., Cowan, N. B., et al. 2009, ApJ, 690, 822

Kuchner, M. 2003, ApJ, 596, 105

Lammer, H., Lichtenegger, H. I. M., Kulikov, Y. N., et al. 2007, Astrobiology, 7, 185

Lammer, H., Odert, P., Leitzinger, M., et al. 2009, A\&\&A, 506, 399

Lederberg, J. 1965, Nature, 207, 9

Léger, A., Selsis, F., Sotin, C., et al. 2004, Icarus, 169, 499

Lovelock, J. E. 1965, Nature, 207, 568

Madhusudhan, N. \& Seager, S. 2009, ApJ, 707, 24

Madhusudhan, N. \& Seager, S. 2010, ApJ, 725, 261

Marley, M. S., Gelino, C., Stephens, D., et al. 1999, ApJ, 513, 879

Miller-Ricci, E., Seager, S., \& Sasselov, D. 2008, ApJ, 690, 1056

Pearl, J. C. \& Christensen, P. R. 1997, JGR 102, 10875

Pilcher, C. B. 2003, Astrobiology, 3, 471

Pont, F., Knutson, H., Gilliland, R. L., et al. 2008, MNRAS, 385, 109

Richardson, L. J., Deming, D., Horning, K., et al. 2007, Nature, 445, 892

Robinson, T. D., Meadows, V., \& Crisp, D. 2010, BAAS 42, 1061

Rogers, L. \& Seager, S. 2010, ApJ, 716, 1208

Rowe, J. F., Matthews, J. M., Seager, S., et al. 2008, ApJ, 689, 1345

Schaefer, L., Fegley, B. 2010, Icarus, 208, 438

Seager, S. \& Sasselov, D. 1998, ApJ, 502, L157

Seager, S. \& Sasselov, D. 2000, ApJ, 537, 916

Seager, S., Whitney, B. A., \& Sasselov, D. D. 2000, ApJ, 540, 504

Seager, S. \& Deming, D. 2010, ARA\& A, 43, 631

Seager, S. 2010, Exoplanet Atmospheres, Physical Processes (Princeton University Press, Princeton)

Seager, S., Schrenk, M., Bains, W., et al. 2011, submitted to Astrobiology

Segura, A., Kasting, J. F. Meadows, V., et al. 2005, Astrobiology, 5, 706

Selsis, F., Kasting, J. F., Levrard, B., et al. 2007, A\&A, 476, 1373

Showman, A. P., Fortney, J. J., Lian, Y., et al. 2009, ApJ, 685, 1324

Showman, A. P. \& Guillot, T. 2002, A\& A, 385, 166

Sudarsky, D., Burrows, A., \& Pinto P. 2000, ApJ, 538, 885

Swain, M. R., Vasisht, G., \& Tinetti, G. 2008, Nature, 452, 329

Swain, M. R., Vasisht, G., Tinetti, G., et al. 2009a, ApJ, 690, L114

Swain, M. R., Tinetti, G., Vasisht, G., et al. 2009b, ApJ, 704, 1616

Swain, M. R., Deroo, P., \& Vasisht, G. 2011, this volume

Turnbull, M. C., Traub, W. A., Jucks, K. W., et al. 2006, ApJ, 644, 551

Vidal-Madjar, A., Lecavelier des Etangs, A., Desert, J.-M., et al. 2003, Nature, 422, 143

von Bloh, W., Bounama, C., Cuntz, M., et al. 2007, A\&\&A, 476, 1365 